\title{
BMJ Global Health Intimate partner violence against women and child maltreatment in a Brazilian birth cohort study: co- occurrence and shared risk factors
}

\author{
Romina Buffarini (D) , ${ }^{1,2}$ Carolina V N Coll (D) , , ${ }^{1,2}$ Terrie Moffitt, ${ }^{4,5}$ \\ Mariângela Freitas da Silveira, ${ }^{1}$ Fernando Barros, ${ }^{1}$ Joseph Murray (D) 1,2
}

To cite: Buffarini R, Coll CVN, Moffitt T, et al. Intimate partner violence against women and child maltreatment in a Brazilian birth cohort study: co-occurrence and shared risk factors. BMJ Global Health 2021;6:e004306. doi:10.1136/ bmjgh-2020-004306

Handling editor Seye Abimbola

Received 28 0ctober 2020 Revised 26 February 2021 Accepted 1 March 2021

Check for updates

(c) Author(s) (or their employer(s)) 2021. Re-use permitted under CC BY. Published by BMJ.

${ }^{1}$ Postgraduate Program in Epidemiology, Department of Social Medicine, Federal University of Pelotas, Pelotas, RS, Brazil

${ }^{2}$ Human Development and Violence Research Centre, Federal University of Pelotas, Pelotas, Brazil

${ }^{3}$ International Center for Equity in Health, Federal University of Pelotas, Pelotas, RS, Brazil

${ }^{4}$ Social, Genetic, and Developmental Psychiatry Centre, Institute of Psychiatry, King's College, London, United Kinkdom

${ }^{5}$ Department of Psychology and Neuroscience, Duke University, Durham, North Carolina, USA

\section{Correspondence to}

Professor Joseph Murray;

j.murray@doveresearch.org

\section{ABSTRACT}

Background Intimate partner violence (IPV) against women and child maltreatment (CM) are major public health problems and human rights issues and may have shared causes. However, their overlap is understudied. We investigated the prevalence of IPV and CM, their cooccurrence in households and possible shared risk factors, in the general population of a Brazilian urban setting. Methods Prospective population-based birth cohort, including over 3500 mother-child dyads with maternal reports on both IPV and CM when children were 4 years old. Eleven neighbourhood, family and parental risk factors were measured between birth and age 4 years. Bivariate and multivariate Poisson regression models with robust variance were used to test which potential risk factors were associated with IPV, CM and their c0-occurrence. Results The prevalence of any IPV and CM were $22.8 \%$ and $10.9 \%$, respectively; the co-occurrence of both types of violence was $5 \%$. Multivariate analyses showed that the overlap of IPV and CM was strongly associated with neighbourhood violence, absence of the child's biological father, paternal antisocial behaviour in general and a mother-partner relationship characterised by high levels of criticism, maternal depression and younger maternal age. A concentration of many risk factors among $10 \%$ of the population was associated with a sixfold increase in risk for overlapping IPV and CM compared with households with no risk factors.

Conclusion IPV and CM share important risk factors in the family and neighbourhood environments and are particularly common in households with multiple social disadvantages and family difficulties. Integrated preventive interventions are needed.

\section{INTRODUCTION}

Violence against women (VAW) and violence against children are two major public health issues. ${ }^{12}$ Worldwide, an estimated one in two children each year suffer some form of violence-such as bullying, violent discipline, emotional abuse, physical abuse, sexual abuse, neglect, fighting or community violence, ${ }^{3}$ and about $30 \%$

\section{Key questions}

What is already known?

- Intimate partner violence (IPV) child maltreatment (CM) are two major public health issues and often occur together inside the same households.

- Evidence on IPV and CM show they are likely to share many risk factors, however, most studies have addressed the two issues separately.

- Studies comparing risk factors for IPV and CM or their overlap are extremely scarce, and no studies assessing the overlap among young children were found.

\section{What are the new findings?}

- The co-occurrence of IPV and CM within the same household was associated with neighbourhood violence, absence of the childs biological father, father antisocial behaviour, a poor mother-partner relationship, maternal depression and maternal young age.

- The concentration of multiple risk factors in the same household was associated with very high risk for IPV, CM and the co-occurrence of both types of violence.

What do the new findings imply?

- This study highlights the need to address violence against women and children as linked phenomena, particularly prevalent in the context of concentrated disadvantage.

- Researchers and policy-makers should aim for greater coordination of prevention programmes tackling IPV and CM.

of women have ever experienced physical partner abuse or sexual violence, ${ }^{4}$ although prevalence can vary substantially by country and survey methodology. ${ }^{35}$ Physical and/ or sexual intimate partner violence (IPV) is estimated to affect about $5 \%-45 \%$ of women each year in different low-income and middle-income countries (LMICs). ${ }^{6}$ The UN Sustainable Development Goals call for increased efforts to eliminate VAW 
and children. Despite the connections between children's and women's experiences of violence, most studies have addressed the two issues independently and overlooked how they often co-occur at home. ${ }^{7}$ Possible shared risks for IPV and child maltreatment (CM) suggest the potential for integrated interventions especially in contexts with high levels of both types of violence. ${ }^{18-10}$

There is significant overlap between IPV and CM in households, ${ }^{11-18}$ and increasing evidence suggests that this has compounding, intergenerational negative effects, both for women and children. ${ }^{19}{ }^{20}$ Separate literatures on IPV and CM show they are likely to share many risk factors, including social norms condoning violence, household poverty and stress, marital conflict and parental use of alcohol and drugs. ${ }^{1}$ However, direct tests comparing risk factors for IPV and CM in the same study-in the same households-are extremely scarce. In a 1985 telephone survey in the USA, $8 \%$ of adults reported that there were both IPV and CM in the home. ${ }^{21}$ Participants reporting only IPV or only CM had many similar characteristics, but co-occurrence was particularly likely in the context of non-violent marital conflict and parental drug use. Two school surveys of Ugandan adolescents ${ }^{20} 22$ found substantial overlap between IPV and CM (present for about one quarter to onethird of the adolescents), and one survey ${ }^{22}$ showed this co-occurrence was associated with lower caregiver education, less emotional attachment in the partner relationship and higher caregiver mental distress and alcohol use. In short, there are very few recent representative studies of the overlap between IPV and CM and possible shared risk factors.

Notably, we found no previous study comparing risk factors for IPV and CM, or their overlap, for young children. Early childhood is a critical period of development, when nurturing care lays essential foundations for healthy outcomes through the life course and exposure to IPV and CM may fundamentally alter children's biology and psychosocial trajectory. ${ }^{23} \mathrm{New}$ studies of IPV, CM, their overlap and determinants are needed, especially in LMICs where household deprivation and violence is so prevalent and especially for young children whose development may be critically affected by exposure to violence.

In Brazil, both IPV and CM are very prevalent and CM severity is unusually high, ${ }^{24-27}$ making it a particularly important context to investigate both types of violence and their co-occurrence. However, only one study to date has investigated the links between IPV and CM in Brazil, in a specific population of 205 women and children (aged 0-18) attending health services in Rio de Janeiro. ${ }^{28}$ Our aim in the current study was to identify the extent of overlap between IPV and CM in a large, population-based birth cohort in southern Brazil and identify independent and shared risk factors for each type of violence.

\section{METHODS}

We conducted a birth cohort study in the city of Pelotas in southern Brazil, with around 340000 inhabitants. In this 2015 Pelotas (Brazil) Birth Cohort Study, eligible children included all hospital-delivered children who were live-born in Pelotas between 1 January and 31 December 2015 and whose mother lived in the urban area of the city. Around 99\% of children born in Pelotas are delivered in hospitals. From the 4333 eligible live births, 4275 were assessed at delivery, equivalent to a response rate of $98.7 \%$. All these children and their mothers were invited to participate in follow-up assessments at 3, 12, 24 and 48 months, with a $95.4 \%$ response rate at the latest assessment at age 48 months. Information used in the current analyses was obtained from mothers (biological or social; non-maternal caregivers were excluded from analyses) using standardised questionnaires. Further information about the 2015 Pelotas Birth Cohort Study is available elsewhere. $^{29}$

\section{Patient and public involvement}

The public was not involved in the design or conduct of our research. Public was involved in the disseminations of the research. Measurements were conducted in confidential interviews and psychological support available when positive responses were given. All participants were informed of the general results through a study newsletter suitable for a non-specialist audience.

\section{Measures of IPV and CM}

IPV and CM were measured in confidential interviews with mothers at the 48-month follow-up in a research centre. Information on IPV was collected using the instrument of the Multi-country Study on Women's Health and Violence Against Women of the WHO. ${ }^{30}$ This instrument asks about acts occurring (yes/no) in the previous 12 months by a current or former partner and has three domains: emotional (four items), physical (six items) and sexual (three items). Each domain is scored positively if at least one of its constituent items is scored 'yes', and a final indicator of any IPV is scored positively if any of the 13 items is scored 'yes'.

$\mathrm{CM}$ was measured using the Portuguese version of the Juvenile Victimization Questionnaire, 2nd edition, Screener Sum Version, Caregiver Lifetime Form (JVQR2). ${ }^{31}$ This is a very widely used instrument internationally, with extensive studies showing that respondents provide reliable and valid data in the context of careful research procedures. ${ }^{32}$ Cross-cultural adaptation and validation in Brazilian population has been conducted previously. ${ }^{33} 34$ In the current study, participants were asked to complete the questionnaire in a private interview with a trained female interviewer at the research centre. Any positive answers indicating possible maltreatment in the child's lifetime instigated an interview with on-site psychologists hired to provide brief counselling and report any cases of current risk of abuse to social services. All participants who were interviewed by psychologists were provided 
with information about appropriate community support services. Based on this questionnaire, the following types of lifetime maltreatment were measured using the Child Maltreatment Module of the JVQ-R2, which includes a single item on each of: physical abuse, emotional abuse, neglect and family abduction/custodial interference; sexual assault by a known adult was also measured using the item in the Sexual Victimisation Module. Each question asks about lifetime victimisation (yes/no). As well as analysing individual types of maltreatment, an indicator of any CM was defined as having ever experienced at least one type of the five types of victimisations listed above. We investigated the possibility of collecting official records of maltreatment, but the number of cases of officially registered maltreatment in the city was extremely small, suggesting such official data would result in nearly all true cases being coded as false negatives and that maternal reports, shown elsewhere to produce high positive response rates, ${ }^{32}$ were the best source of information in this study.

\section{Measures of possible risk factors}

The following were considered as possible risk factors for IPV and CM and their overlap: neighbourhood violence, family income, maternal and paternal education and age, father presence/absence, father antisocial behaviour, mother-father relationship quality, maternal depression, alcohol use and illicit drug use. These possible risk factors were chosen a priori based on reviews of risk factors for $\mathrm{IPV},{ }^{35} \mathrm{CM}^{36-38}$ and, in particular, reviews of probable risk factors for their co-occurrence. ${ }^{139}$

Neighbourhood violence was assessed at the 48-month follow-up using questions ${ }^{40}$ scored $0-3$ (never to often) about the frequency of four violent acts in the participant's neighbourhood in the last 6 months: fights with weapons, fights between gangs, robbery and sexual violence. The scores were summed and then split into three categories, indicating increasing levels of violence $(0-2,3-7$ and $8-12)$.

Information on family income, parental schooling and age was collected during the perinatal assessment. Family income, collected as a continuous variable, was obtained by summing the monthly income of all household members and then dividing the total into quintiles. Parents' schooling was measured in complete years of formal education and then categorised into four groups $(0-4,5-8,9-11$ and $\geq 12)$. Mother's and father's age in complete years was classified as $<20,20-33$ and $\geq 35$.

Mothers provided information on whether the child's father was living with the child at the 48-month assessment and specified whether the biological, adoptive or social father lived at home. The final variable used in the current analyses had three categories: (1) no father at home, (2) biological father at home and (3) social father at home. Twenty-four children with adopted fathers were excluded from this analysis, given this small number would not permit separate analysis, and combining adoptive fathers who are carefully screened by adoption agencies, with other social fathers, would not be appropriate. The category 'no father at home' means that no father figure (either biological or social) is living at home, even though the mother may have a partner outside the home.

Father antisocial behaviour was evaluated using the Antisocial Personality Module of the Mini International Neuropsychiatric Interview (MINI). ${ }^{41-43}$ Usually the MINI is used to measure the respondent's own psychiatric symptoms, but in this study, mothers were asked about the behaviour of the child's father-either biological or social, whoever had most contact with the child. A previous study by Caspi et al showed that mothers' reports about paternal antisocial behaviours were reliable and valid. ${ }^{44}$ For the current analyses, we created a total father antisocial behaviour score by summing five of the questions (yes/no), but excluding the sixth question on domestic violence, in order to test the extent to which fathers' general antisocial behaviour was a risk factor for IPV and CM. Fathers scoring 1+ were categorised as antisocial.

Mother-partner relationship conflict was assessed at 3 months, using two Likert-type questions ${ }^{45}$ about partners' criticism of each other, ranging from 1 to 10 (low to high criticism). Each question asked how critical the mother considered her partner of her and how critical she considered herself to be of her partner. We first categorised each individual score as reflecting low (1-3), medium (4-6) or high (7-10) criticism, and then combined the two into a relationship criticism score coded as follows: low criticism (low-low or low-medium), medium criticism (mediummedium or low-high) or high criticism (medium-high or high-high).

Maternal depression was defined as scoring 13 or more points on the Edinburgh Postnatal Depression Scale, applied at 3 months, and previously validated in the same population. ${ }^{46}$ Finally, maternal alcohol drinking and drug use was assessed at 48 months with the Alcohol, Smoking and Substance Involvement Screening Test. ${ }^{478}$ Drug use was characterised as use of any illicit substance in the 3 months prior to interview, and alcohol use was defined as daily drinking of any alcoholic beverage.

\section{Analyses}

The first set of analyses examined the prevalence and co-occurrence of IPV and CM. For twins, one sibling was excluded $(n=48)$ and for one case of triplets, two siblings were excluded $(n=2)$, so as to not count IPV against one mother multiple times. In the first descriptive analysis of individual outcomes, participants with valid data on each separate outcome $(\mathrm{CM}, \mathrm{n}=3723$; IPV $\mathrm{n}=3533$ ) were included. For subsequent analyses of co-occurrence, mother-child pairs with complete information $(n=3533)$ on the two outcomes-IPV and CM-were included. Co-occurrence is first visually represented in a Venn diagram. The extent of observed co-occurrence was compared with the statistically expected probability of co-occurrence due to chance (assuming that the two 
forms of violence are independent of each other). The expected prevalence of co-occurrence was estimated by multiplying the proportion of women who have experienced IPV by the proportion of children who experienced maltreatment. To test the significance of the difference between observed and expected co-occurrence, we compared the $95 \%$ CIs of the observed and expected prevalence rates. The observed and expected co-occurrence rates were reported as statistically different when the lower limit of the observed co-occurrence prevalence $95 \%$ CI was above the upper limit of the $95 \%$ CI for the expected co-occurrence. The $95 \%$ CI was calculated using the variance for the expected co-occurrence, defined as follows: var IPV $-\mathrm{CM}=\operatorname{var} \mathrm{IPV} \times \operatorname{var} \mathrm{CM}+\operatorname{var} \mathrm{IPV} \times(\mathrm{ECM}) 2+\operatorname{var} \mathrm{CMt} \times(\mathrm{E} I P V) 2$, where $\mathrm{E}=$ estimate and $\operatorname{Var}=\mathrm{SE}^{2}(\mathrm{n})$.

In the second set of analyses, IPV and CM were considered in relation to possible risk factors. For these analyses, in order to consider whether IPV and CM share risk factors, three separate outcomes were examined: only IPV (no CM reported), only CM (no IPV reported) and co-occurrence of both types of violence. For each of the three outcome variables, the reference group is the rest of the sample (eg, for the only IPV outcome variable, the reference group includes those experiencing no violence, only CM and co-occurrence). Prevalence ratios and 95\% CI for the crude and adjusted associations between risk factors and violence outcomes were estimated using Poisson regression with robust variance. ${ }^{49}$

In adjusted analyses, risk factors were considered in seven hierarchal levels. The first level included neighbourhood violence. Levels 2-4 included the following socioeconomic and demographic variables: maternal and paternal age (level 2), maternal and paternal education (level 3) and family income (level 4). In the fifth level, father's antisocial behaviour was added, and in the sixth level, father cohabitation with the child. The seventh level included behavioural, relationship and health characteristics that could have bidirectional associations. Therefore, when analysing the seventh level, separate models were estimated for each risk factor, without adjusting for other variables in the seventh level: mother-partner relationship (model 7a), maternal depression (model 7b), maternal alcohol use (model 7c) and maternal drug use (model $7 \mathrm{~d}$ ). The rationale for this adjusted analysis, adjusting for variables by hierarchical order, was to avoid adjusting for variables likely to represent mediating mechanisms between each risk factor and outcome ${ }^{50}$ Variables with $\mathrm{p}<0.20$ were kept in the model as possible confounders for subsequent levels. For levels 2 and 3, relevant variables were entered simultaneously in the adjusted analyses. Correlations between the variables were low (online supplemental table 1) and did not cause multicollinearity problems in the models.

Finally, the proportion of participants with each outcome was examined according to a cumulative risk score. For these analyses, first, each risk factor was dichotomised to compare the category representing the highest risk to all other categories. With this coding of 1 or 0 for
Table 1 Prevalence of intimate partner violence (IPV) against women and child maltreatment (CM) in the 2015 Pelotas (Brazil) Birth Cohort Study

\begin{tabular}{lrc}
\hline & N & \% (95\% Cl) \\
\hline Any IPV against women & 804 & $22.8(21.4$ to 24.2$)$ \\
\hline Emotional violence & 766 & $21.7(20.4$ to 23.1$)$ \\
\hline Physical violence & 262 & $7.5(6.6$ to 8.3$)$ \\
\hline Sexual violence & 55 & $1.6(1.2$ to 2.0$)$ \\
\hline Any CM & 405 & $10.9(9.9$ to 11.9$)$ \\
\hline Psychological/emotional abuse & 290 & $7.8(7.0$ to 8.7$)$ \\
\hline $\begin{array}{l}\text { Physical abuse } \\
\text { Neglect }\end{array}$ & 62 & $1.7(1.3$ to 2.1$)$ \\
\hline $\begin{array}{l}\text { Family abduction/custodial } \\
\text { interference }\end{array}$ & 74 & $1.7(1.3$ to 2.2$)$ \\
\hline Sexual assault by known adult & 9 & $0.2(0.1$ to 0.5$)$ \\
\hline
\end{tabular}

Total number of mothers with valid data for IPV: 3533.

Total number of children with valid data for maltreatment: 3723 .

every risk factor, a cumulative risk score was then calculated by summing across risk factors, and outcomes were examined according to this cumulative risk score.

Analyses were carried out in STATA V.14 (StataCorp, College Station, USA).

\section{RESULTS}

Information on IPV and CM was collected for 3533 mothers and 3723 children, respectively, representing $82.6 \%$ and $87.1 \%$ of the original participants in the cohort recruited at birth. The mean age of children was 45.5 months $(\mathrm{SD}=2.6)$ and $50.6 \%$ were boys $(\mathrm{N}=1886)$. Table 1 shows rates of overall IPV and overall CM in the study, as well as their individual subtypes. Overall, $22.8 \%$ of mothers reported some form of IPV in the previous year. The most prevalent form of IPV was emotional violence $(21.7 \%)$, followed by physical violence $(7.5 \%)$ and sexual violence $(1.6 \%)$. Regarding CM, $10.9 \%$ of children were reported to have experienced any form of maltreatment in the child's lifetime. Children were most commonly exposed to emotional abuse $(7.8 \%)$, followed by family abduction/custodial interference, neglect and physical abuse (each with a prevalence of about $2 \%$ ) and sexual assault by a known adult $(0.2 \%)$. Comparing each form of violence between girls and boys, the only difference observed was a slightly elevated rate of overall $\mathrm{CM}$ for boys ( $11.9 \%$ for boys vs $9.8 \%$ for girls; online supplemental table 2).

Figure 1 shows the extent to which IPV and CM overlapped within households or occurred in isolation. For $4.6 \%$ of the study population, there was an overlapmothers reported both IPV and CM. This observed rate of co-occurrence is twice the rate compared with that which would be expected by chance $(2.4 \%-95 \%$ CI 2.0 to 2.8), given the overall rates of IPV and CM in the study population $(\mathrm{p}<0.05$ for the difference between observed 


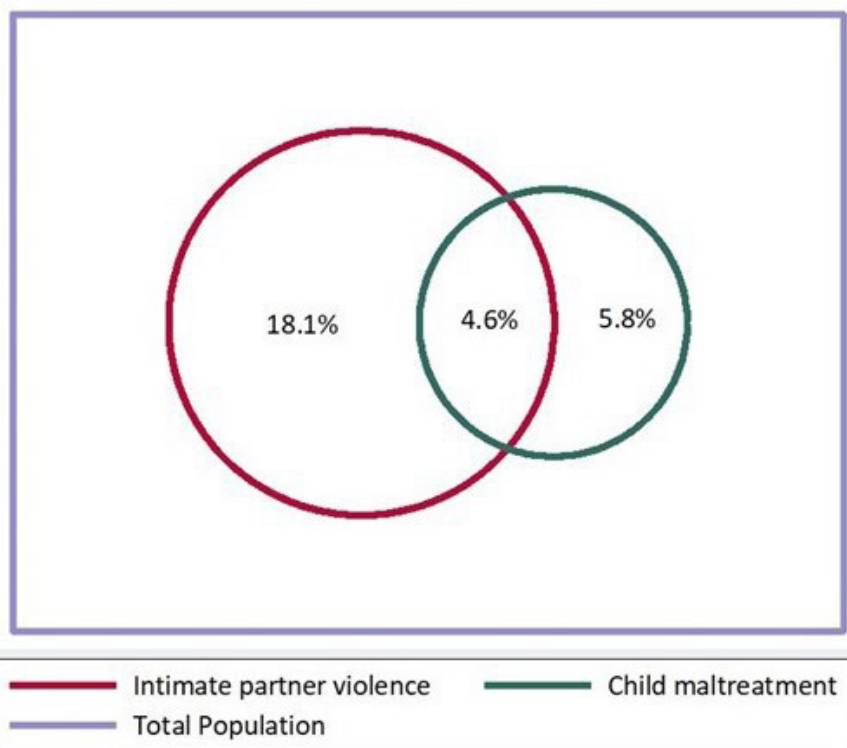

Figure 1 Venn diagram of intimate partner violence against women and child maltreatment in the 2015 Pelotas Birth Cohort $(\mathrm{N}=3533)$.

and expected rates). This difference suggests that there are likely to be shared risk factors for IPV and CM.

Table 2 shows the rates of IPV only, CM only and their co-occurrence, according to family and parental characteristics and levels of neighbourhood violence. Five family and parent characteristics were strongly and significantly associated with all three violence outcomes: low family income, biological father not living with the child, father antisocial behaviour, poor mother-partner relationship and maternal depression. The following were associated with IPV and its co-occurrence with CM, but were not associated with CM on its own: neighbourhood violence, low paternal education and maternal use of illicit drugs. For CM on its own and in co-occurrence with IPV, there were strong associations with low maternal education and young age of the mother, but these risk factors did not associate with IPV alone.

Table 3 shows associations between risk factors and the three violence outcomes, adjusting for other risk factors according to the hierarchical model. Nearly all risk factors were independently associated with violence against both women and children, even after controlling for confounders. The risk factors that were associated with all three violence outcomes (IPV only, CM only and their co-occurrence) were: biological father not living at home, father antisocial behaviour, poor maternalpartner relationship and maternal depression.

Summing women and children's exposure across all the risk factors (based on the results in table 3, the 'high risk' categories chosen to calculate these 'cumulative risk scores' were as follows (high risk in brackets): neighbourhood violence (8-12); paternal age $(<20)$; maternal age $(<20)$; maternal education $(0-4)$; paternal education (0-4); family income (lowest quintile); father antisocial (yes); father lives with child (no father at home); mother-partner relationship (medium-high criticism); maternal depression (yes); maternal alcohol (yes) and maternal drugs (yes)), cumulative risk scores in the population ranged from 0 to $8(\mathrm{~m}=1.4 ; \mathrm{SD}=1.4)$. Of all households, $34.9 \% \quad(\mathrm{n}=1303)$ had no risk factors; 26.5\% (n 990) had one risk factor; $17.9 \%(\mathrm{n}=669)$ had two risk factors; $10.8 \%(\mathrm{n}=402)$ had three risk factors and $9.9 \%(\mathrm{n}=366)$ had four or more risk factors. As figure 2 shows, there was a linear trend between the number of risk factors in the household and the likelihood of each violent outcome (IPV only, CM only as well as their co-occurrence). Among households with 4+ risk factors, one in two women reported either or both IPV and CM $(30 \%$ of women experienced IPV only, $10 \%$ of children experienced CM only and $11 \%$ of households suffered both IPV and CM). Comparing households with 4+ risk factors to those with none, the increased risk for IPV only was 2.8 (95\% CI 2.2 to 3.5, $\mathrm{p}<0.001$ ); the increased risk for $\mathrm{CM}$ only was 2.6 (95\% CI 1.7 to $4.0, \mathrm{p}<0.001)$ and the risk of co-occurring IPV and CM was raised over sixfold $(\mathrm{PR}=6.4$, $95 \%$ CI 3.8 to $10.6, \mathrm{p}<0.001)$.

\section{DISCUSSION}

As far as we are aware, this is the first population-based study of young children to investigate risk factors for CM and maternal IPV and their overlap. Rates of IPV (22.8\%) and CM (10.9\%) in our study were high, although not as high as some other studies have reported, ${ }^{3-651}$ possibly due to heterogeneity in survey methodologies, participant age, settings, measures and definitions of violence. Some $5 \%$ of families experienced both forms of violence by the time children were 4 years old in our Brazilian setting. Risk factors contributing to the co-occurrence of IPV and CM included residing in a violent neighbourhood, absence of the biological father, father antisocial behaviour, poor mother-partner relationship, maternal depression and maternal young age. The concentration of multiple risk factors in the same household was associated with very high risk for all three violence outcomes: IPV only, CM only and the co-occurrence of both IPV and CM.

Our estimate that $5 \%$ of mother-child pairs experienced both IPV and CM is hard to compare with previous studies that have included measures of both, given the range of samples, types of violence examined (particularly for $\mathrm{CM}$ ), reference periods and instruments used. ${ }^{39} 52$ However, uniformly studies have reported an increased risk for CM in the context of IPV..$^{11-14} 161820$ In a previous survey in Brazil, the co-occurrence of physical IPV and CM was $12.2 \%$ in a small sample of women and children attending health services in the city of Rio de Janeiro and was selected for a case-control study about domestic violence and premature birth. ${ }^{28}$ Across the global south, we could find only two previous studies of factors associated with the co-occurrence of IPV and CM, both in Uganda. One study of over 3000 Ugandan adolescents found that the combination of witnessing IPV and 
Table 2 Intimate partner violence against women, child maltreatment and co-occurrence of both types of violence according to family and neighbourhood characteristics

\begin{tabular}{|c|c|c|c|c|}
\hline & Total N & $\%$ only IPV & $\%$ only CM & $\%$ co-occurrence \\
\hline Neighbourhood violence & & $P=0.005$ & $P=0.394$ & $P=0.005$ \\
\hline $0-2$ & 2057 & 16.7 & 5.4 & 3.7 \\
\hline $3-7$ & 1196 & 19.3 & 6.6 & 5.6 \\
\hline $8-12$ & 279 & 24.0 & 6.1 & 7.2 \\
\hline Family income (quintiles) & & $P<0.001$ & $P=0.078$ & $P=0.035$ \\
\hline Q1 (poorest) & 659 & 24.0 & 5.9 & 6.2 \\
\hline Q2 & 741 & 18.9 & 7.8 & 5.5 \\
\hline Q3 & 716 & 18.9 & 5.9 & 4.5 \\
\hline Q4 & 721 & 15.8 & 5.4 & 3.6 \\
\hline Q5 (richest) & 694 & 13.5 & 4.3 & 3.2 \\
\hline Maternal education (years) & & $P=0.154$ & $P=0.001$ & $P=0.001$ \\
\hline $0-4$ & 298 & 19.8 & 10.7 & 7.1 \\
\hline $5-8$ & 893 & 19.8 & 5.6 & 6.5 \\
\hline $9-11$ & 1244 & 18.3 & 6.2 & 3.7 \\
\hline 12 or more & 1097 & 16.1 & 4.5 & 3.5 \\
\hline Maternal age (years) & & $P=0.839$ & $P=0.135$ & $P=0.032$ \\
\hline$<20$ & 499 & 18.6 & 7.8 & 6.6 \\
\hline 20-34 & 2524 & 17.9 & 5.5 & 4.5 \\
\hline$\geq 35$ & 509 & 18.9 & 5.9 & 3.1 \\
\hline Paternal education (years) & & $P=0.001$ & $P=0.488$ & $P=0.036$ \\
\hline $0-4$ & 445 & 19.3 & 6.3 & 6.3 \\
\hline $5-8$ & 966 & 22.1 & 5.7 & 5.2 \\
\hline $9-11$ & 1089 & 17.5 & 6.0 & 3.6 \\
\hline$\geq 12$ & 854 & 14.9 & 4.6 & 3.5 \\
\hline Paternal age (years) & & $P=0.644$ & $P=0.118$ & $P=0.862$ \\
\hline$<20$ & 194 & 20.6 & 8.8 & 4.6 \\
\hline $20-34$ & 2360 & 17.9 & 6.0 & 4.4 \\
\hline$\geq 35$ & 939 & 18.2 & 5.0 & 4.8 \\
\hline Father lives with child & & $\mathrm{P}<0.001$ & $P<0.001$ & $P<0.001$ \\
\hline No father at home & 717 & 24.9 & 9.5 & 8.4 \\
\hline Social father at home & 187 & 13.9 & 17.1 & 8.6 \\
\hline Biological father at home & 2627 & 16.3 & 4.1 & 3.3 \\
\hline Father antisocial behaviour & & $\mathrm{P}<0.001$ & $P=0.003$ & $\mathrm{P}<0.001$ \\
\hline Yes & 683 & 35.0 & 8.4 & 11.6 \\
\hline No & 2789 & 13.9 & 5.2 & 2.8 \\
\hline Mother-partner relationship & & $\mathrm{P}<0.001$ & $P=0.005$ & $\mathrm{P}<0.001$ \\
\hline Low criticism & 1975 & 14.1 & 4.0 & 2.8 \\
\hline Low-to-medium criticism & 596 & 23.3 & 6.2 & 6.0 \\
\hline Medium-to-high criticism & 426 & 29.8 & 7.3 & 6.8 \\
\hline Maternal depression & & $\mathrm{P}<0.001$ & $P=0.001$ & $\mathrm{P}<0.001$ \\
\hline No & 3113 & 17.0 & 5.3 & 3.7 \\
\hline Yes & 361 & 27.4 & 10.0 & 11.6 \\
\hline Daily maternal use of alcohol & & $P=0.821$ & $P=0.125$ & $P=0.420$ \\
\hline No & 2805 & 19.2 & 5.6 & 5.2 \\
\hline
\end{tabular}


Table 2 Continued

\begin{tabular}{lclcc}
\hline & Total N & \% only IPV & \% only CM & \% co-occurrence \\
\hline Yes & 34 & 17.7 & 11.8 & 8.8 \\
Maternal use of illicit drugs & & $\mathrm{P}<0.001$ & $\mathrm{P}=0.167$ & $\mathrm{P}=0.075$ \\
No & 3409 & 17.7 & 5.8 & 4.5 \\
Yes & 123 & 30.9 & 8.9 & 8.1 \\
\hline
\end{tabular}

Wald test $p$ value.

Row percentages.

All variables have $<5 \%$ missing data, except daily maternal use of alcohol (20\%) and mother-partner relationship (17.5\%).

Co-occurrence of any IPV and CM.

$\mathrm{CM}$, child maltreatment; IPV, intimate partner violence against mother.

experiencing violence was associated with outcomes such as mental health problems, but was not associated with demographic characteristics of the household. ${ }^{20}$ The second study, including around 300 Ugandan adolescents, found that overlapping IPV and CM was associated with lower caregiver education and SES (socioeconomic status), lower partner attachment and higher mental distress and alcohol use among female caregivers and lower partner attachment as well as attitudes favouring violence against children among male caregivers. ${ }^{22}$ No studies of risk factors for IPV and CM among young children were found.

The current study empirically confirms, in a large population-based birth cohort, the theoretical proposition, derived principally from two parallel literatures, that IPV and CM share many risk factors. The shared risk factors in this Brazilian study point to the relevance of neighbourhood, family structure and household circumstances in increasing the risk for IPV, CM as well as the wider social and cultural influences implied by an ecological understanding of violence. Common aetiologies of IPV and CM involve stressful environments, problematic family relationships and male antisocial behaviour. Especially notable in the current study, elevated rates of both IPV and CM were found among families characterised by the absence of a biological father and by partner conflict, as has been reported previously in studies of IPV or CM alone. ${ }^{19}$ Social fathers have been found to perpetrate $\mathrm{CM}$ at higher rates than biological fathers, ${ }^{53} 54$ possibly because of lower attachment to non-genetically related children and related difficulties in developing roles as coparents. ${ }^{55}$ The presence of a stepfather was also associated with increased risk of child physical abuse in another Brazilian study. ${ }^{56}$ However, the benefits of living with two biological parents seems highly dependent on the quality of parental care and in particular levels of paternal antisocial behaviour. ${ }^{57}$ In the current study, paternal antisocial behaviour in general (ie, not necessarily directed at the mother or child) increased risk for both IPV and $\mathrm{CM}$ and their co-occurrence. Thus, difficult relationships with antisocial males, who are not necessarily children's fathers, seem the most important risk factors for joint IPV and $\mathrm{CM}$ in this study. Association with violent men, while never the mother's fault, has been understood to partly arise in the context of women's own experiences of childhood violence, and maternal depression (also identified as a risk factor in our study) is considered one pathway to be addressed in the intergenerational transmission of $\mathrm{CM}^{58}$

Critically, within some households there was a marked concentration of risk factors for family violence, with implications for intergenerational transmission. ${ }^{59}$ Exposure to multiple risk factors conferred increasing risk for both IPV and CM in a linear fashion. For the $10 \%$ of households with four or more risk factors, the likelihood of VAW and children was exceptionally high-half of mothers in such households reported IPV, CM or both, and co-occurring IPV and CM was six times more likely to occur for them than in households with no risk factors. In such vulnerable circumstances, individuals and families thus often experience multiple forms of violence that may be extremely difficult to address in isolation and without considerable support to face multiple family challenges. Although universal prevention programmes are important to alter social norms associated with violence and reduce risk factors across the population, intensive work with particularly vulnerable families, such as through multisystemic therapy with intersectoral support, is thus also critical to stem the intergenerational transmission of violence. In the current study, poorer, teenage and less educated mothers were at heightened risk for co-occurring IPV and CM and thus targeted interventions for younger disadvantaged women seem appropriate. Nurse home visiting programmes, such as the Nurse-Family partnership, have good evidence for reducing $\mathrm{CM}$ among young, disadvantaged women, and there is some evidence that this type of intervention may also reduce IPV. ${ }^{60}$

This study shows the need for both primary and secondary prevention strategies to reduce family violence, as well as treatment, especially in multipleproblem households where IPV and CM are most likely to co-occur. The exposure of young children to violence, both as victims of maltreatment and as witnesses to violence between adults, highlights the need for a lifecourse perspective, with longitudinal studies elucidating at which stages of development children are most exposed to violence and the optimum periods for 
Table 3 Adjusted associations between potential risk factors and IPV, CM and the co-occurrence of both types of violence

\begin{tabular}{|c|c|c|c|c|}
\hline \multirow[b]{2}{*}{ Level } & \multirow[b]{2}{*}{ Variable } & \multirow{2}{*}{$\begin{array}{l}\text { Only IPV } \\
\text { PR (95\% CI) }\end{array}$} & \multirow{2}{*}{$\begin{array}{l}\text { Only CM } \\
\text { PR (95\% Cl) }\end{array}$} & \multirow{2}{*}{$\begin{array}{l}\text { Co-occurrence } \\
\text { PR }(95 \% \mathrm{Cl})\end{array}$} \\
\hline & & & & \\
\hline \multirow[t]{3}{*}{1} & Neighbourhood violence score & $P=0.004$ & $P=0.395$ & $P=0.005$ \\
\hline & $3-7$ & $1.2(1.0$ to 1.3$)$ & $1.2(0.9$ to 1.6$)$ & $1.5(1.1$ to 2.1$)$ \\
\hline & $8-12$ & $1.4(1.1$ to 1.8$)$ & $1.1(0.7$ to 1.8$)$ & $1.9(1.2$ to 3.1$)$ \\
\hline \multirow{5}{*}{2} & $<20$ & $1.1(0.8$ to 1.6$)$ & $1.6(0.8$ to 3.0$)$ & $0.5(0.2$ to 1.1$)$ \\
\hline & $20-34$ & $1.0(0.8$ to 1.2$)$ & $1.4(0.9$ to 1.8$)$ & 0.7 (0.5 to 1.0$)$ \\
\hline & $\geq 35$ & Reference & Reference & Reference \\
\hline & Maternal age (years) & $P=0.894$ & $P=0.396$ & $P=0.003$ \\
\hline & $<20$ & $0.9(0.7$ to 1.3$)$ & $1.0(0.6$ to 1.8$)$ & $2.8(1.5$ to 5.4$)$ \\
\hline \multirow{8}{*}{3} & $0-4$ & $0.9(0.7$ to 1.2$)$ & 2.5 (1.3 to 4.3$)$ & 1.7 (0.9 to 3.4$)$ \\
\hline & $5-8$ & $1.0(0.8$ to 1.3$)$ & $1.3(0.8$ to 2.1$)$ & $1.4(0.8$ to 2.4$)$ \\
\hline & $9-11$ & $1.0(0.8$ to 1.2$)$ & $1.3(0.8$ to 1.9$)$ & $0.9(0.6$ to 1.5$)$ \\
\hline & 12 or more & Reference & Reference & Reference \\
\hline & Paternal education (years) & $P=0.021$ & $P=0.765$ & $P=0.858$ \\
\hline & $0-4$ & $1.3(0.9$ to 1.7$)$ & $0.9(0.5$ to 1.6$)$ & 1.1 (0.6 to 2.1$)$ \\
\hline & $5-8$ & $1.4(1.1$ to 1.8$)$ & 0.9 (0.6 to 1.5$)$ & 1.0 (0.6 to 1.8$)$ \\
\hline & $9-11$ & $1.1(0.9$ to 1.4$)$ & $1.1(0.7$ to 1.7$)$ & $0.9(0.6$ to 1.4$)$ \\
\hline 4 & Q5 (richest) & Reference & Reference & Reference \\
\hline \multirow[t]{3}{*}{5} & Father antisocial behaviour & $P<0.001$ & $P=0.019$ & $P<0.001$ \\
\hline & Yes & $2.4(2.1$ to 2.8$)$ & $1.5(1.1$ to 2.0$)$ & 3.9 (2.9 to 5.5$)$ \\
\hline & No & Reference & Reference & Reference \\
\hline \multirow[t]{4}{*}{6} & Father lives with child & $P=0.048$ & $\mathrm{P}=<0.001$ & $P=0.038$ \\
\hline & No father at home & $1.2(1.0$ to 1.4$)$ & 2.1 (1.4 to 2.9 ) & 1.4 (1.0 to 2.2$)$ \\
\hline & Social father at home & 0.7 (0.5 to 1.1$)$ & 3.5 (2.3 to 5.4$)$ & $2.0(1.1$ to 3.8$)$ \\
\hline & Biological father at home & Reference & Reference & Reference \\
\hline \multirow[t]{4}{*}{$7 a$} & Mother-partner relationship & $P<0.001$ & $P<0.001$ & $P=0.013$ \\
\hline & Low criticism & Reference & Reference & Reference \\
\hline & Low-to-medium criticism & $1.5(1.2$ to 1.8$)$ & $1.5(1.2$ to 1.8$)$ & 1.7 (1.1 to 2.7$)$ \\
\hline & Medium-to-high criticism & 1.9 (1.5 to 2.2$)$ & 1.9 (1.5 to 2.3$)$ & 1.8 (1.1 to 2.8$)$ \\
\hline \multirow[t]{3}{*}{$7 b$} & Maternal depression & $\mathrm{P}<0.001$ & $P=0.007$ & $P<0.001$ \\
\hline & No & Reference & Reference & Reference \\
\hline & Yes & $1.5(1.2$ to 1.8$)$ & 1.7 (1.2 to 2.5$)$ & $2.4(1.6$ to 3.6$)$ \\
\hline
\end{tabular}

Continued 
Table 3 Continued

\begin{tabular}{|c|c|c|c|c|}
\hline \multirow[b]{2}{*}{ Level } & \multirow[b]{2}{*}{ Variable } & \multirow{2}{*}{$\begin{array}{l}\text { Only IPV } \\
\text { PR (95\% CI) }\end{array}$} & \multirow{2}{*}{$\begin{array}{l}\text { Only CM } \\
\text { PR (95\% CI) }\end{array}$} & \multirow{2}{*}{$\begin{array}{l}\text { Co-occurrence } \\
\text { PR }(95 \% \mathrm{Cl})\end{array}$} \\
\hline & & & & \\
\hline \multirow[t]{3}{*}{$7 c$} & Maternal use of alcohol & $P=0.109$ & $P=0.109$ & $P=0.671$ \\
\hline & No & Reference & Reference & Reference \\
\hline & Yes & $0.4(0.2$ to 1.2$)$ & $2.1(0.8$ to 5.4$)$ & $1.3(0.4$ to 4.2$)$ \\
\hline \multirow[t]{3}{*}{$7 d$} & Maternal use of illicit drugs & $P=0.065$ & $P=0.521$ & $P=0.339$ \\
\hline & No & Reference & Reference & Reference \\
\hline & Yes & $1.3(0.9$ to 1.8$)$ & $1.2(0.6$ to 2.3$)$ & $1.4(0.7$ to 2.6$)$ \\
\hline
\end{tabular}

Variables are adjusted for variables in all previous levels as well as other variables in the same level-except for variables in the seventh level, which are not adjusted for each other.

For each of the three outcome variables, the reference group is the rest of the sample (eg, for the only IPV outcome variable, the reference group includes those experiencing no violence, only $\mathrm{CM}$ and co-occurrence).

Co-occurrence of IPV and CM.

CM, child maltreatment; IPV, intimate partner violence against women; PR, prevalence ratio.

intervention considering different possible determinants as children age.

This study has important strengths, including its urban population-based design, large sample, high follow-up rates, in-person interviews by trained research staff providing more reliable data on IPV and CM than official records and a wide range of possible risk factors in a longitudinal cohort. The findings should also be interpreted considering limitations of the study. First, results should not be generalised to rural populations. Second, as with all self-report studies of violence, there is likely to be under-reporting of violence in the current study. Third, there is possible reporter bias, as all information was provided by mothers, and this could contribute to overestimating associations. Reports on IPV or CM might also be biased by maternal mental health-and the association between violence and maternal depression in particular might be biased by using maternal reports only. Lastly, reverse causation is possible because many risk factors were measured simultaneously with family violence. For example, father absence could be a consequence of family violence, rather than a

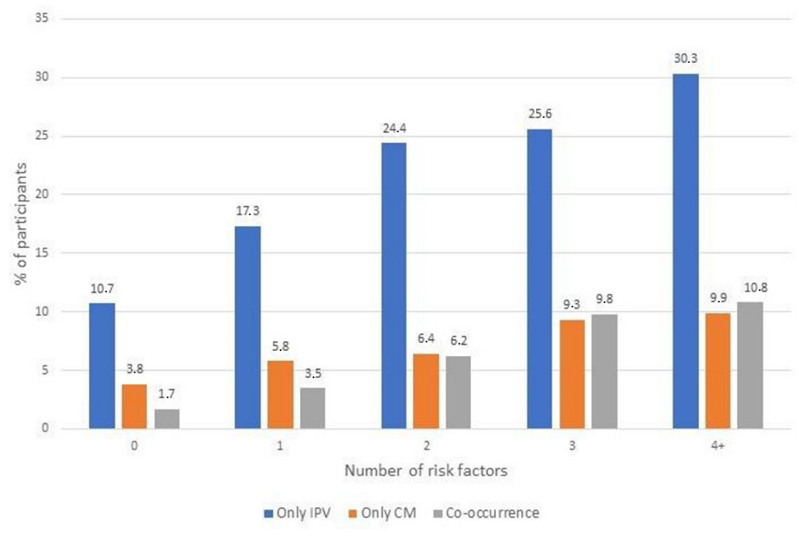

Figure 2 Prevalence of each of each type of violence outcome according to the number of risk factors in the household. CM, child maltreatment; IPV, intimate partner violence. prospective risk factor. To minimise this bias, with IPV and CM as outcomes measured when children were age 4 years, wherever possible we selected measures of risk factors assessed previously in the cohort (eg, measures of partner relationship quality and maternal depression at 3 months postpartum); nonetheless, this does not rule out reverse causality. Bidirectional associations are likely to exist and this was considered in our hierarchical model, but we are unable to establish causal relationships in this study. Another limitation is that the current study did not identify the perpetrators of family violence.

\section{CONCLUSION}

A false view that focusing on the problem of violence against children could undermine addressing VAW (and vice versa) has resulted in historically parallel but distinctive advocacy, research and response efforts. This study provides critical evidence that IPV and CM are prevalent and overlapping issues with several common risk factors and emphasises the need to address VAW and children as a joint phenomenon arising particularly in the context of concentrated disadvantage. Researchers and policymakers should aim for greater coordination between IPV and CM prevention programmes to advance both fields in the best interests of women and children.

Twitter Romina Buffarini @RominaBuffarini

Acknowledgements The authors are grateful to all the families who took part in the 2015 Pelotas Birth Cohort and the Pelotas teams, including research scientists, interviewers, workers and volunteers.

Contributors RB and JM designed the study. RB performed the analysis. RB, CVNC, JM and TM interpreted the results. MFdS, FB and JM participated in the design and conduct of the original cohort study and critical reviewing of the manuscript. CVNC, RB and JM wrote the manuscript. TM critically reviewed the manuscript. All authors contributed to and approved the final version.

Funding This article is based on data from the study 'Pelotas Birth Cohort, 2015' conducted by Postgraduate Program in Epidemiology at Universidade Federal de Pelotas, with the collaboration of the Brazilian Public Health Association (ABRASCO). The first phases of the 2015 Pelotas (Brazil) Birth Cohort were funded by the Wellcome Trust (095582). Funding for specific follow-up visits was also received from the Conselho Nacional de Desenvolvimento Científico e Tecnológico (CNPq) and Fundação de Amparo à Pesquisa do Estado do Rio Grande do Sul 
(FAPERGS) and Children's Pastorate sponsored follow-up at 24 months and FAPERGS-PPSUS (Programa de Pesquisa para o Sistema Único de Saúde), the Wellcome Trust (10735_Z_18_Z) and the Bernard van Leer Foundation (BRA2018-178) for the 48 months follow-up. RB is supported by Departamento de Ciências e Tecnologia.

\section{Competing interests None declared.}

Patient consent for publication Not required.

Ethics approval The cohort study was approved by the Ethics Committee of the School of Physical Education, Federal University of Pelotas (CAAE registration number: 26746414.5.0000.5313), age 48-month psychosocial assessments, including assessments of violence that were approved by the Ethics Committee of the Faculty of Medicine, Federal University of Pelotas (CAAE registration number: 03837318.6.0000.5317) and written informed consent was obtained from parents or guardians at each visit.

\section{Provenance and peer review Not commissioned; externally peer reviewed.}

Data availability statement Data are available upon request. Due to confidentiality restrictions related to the ethics approval for this study, no identifying information about participants may be released. Dataset without identification used during the current study is available from the corresponding author on reasonable request.

Supplemental material This content has been supplied by the author(s). It has not been vetted by BMJ Publishing Group Limited (BMJ) and may not have been peer-reviewed. Any opinions or recommendations discussed are solely those of the author(s) and are not endorsed by BMJ. BMJ disclaims all liability and responsibility arising from any reliance placed on the content. Where the content includes any translated material, BMJ does not warrant the accuracy and reliability of the translations (including but not limited to local regulations, clinical guidelines, terminology, drug names and drug dosages), and is not responsible for any error and/or omissions arising from translation and adaptation or otherwise.

Open access This is an open access article distributed in accordance with the Creative Commons Attribution 4.0 Unported (CC BY 4.0) license, which permits others to copy, redistribute, remix, transform and build upon this work for any purpose, provided the original work is properly cited, a link to the licence is given, and indication of whether changes were made. See: https://creativecommons.org/ licenses/by/4.0/.

\section{ORCID iDs}

Romina Buffarini http://orcid.org/0000-0002-6905-8767

Carolina V N Coll http://orcid.org/0000-0003-0808-8230

Joseph Murray http://orcid.org/0000-0002-5511-3454

\section{REFERENCES}

1 Guedes A, Bott S, Garcia-Moreno C, et al. Bridging the gaps: a global review of intersections of violence against women and violence against children. Glob Health Action 2016;9:31516.

2 Patel DM. Preventing violence against women and children: workshop summary, 2011. doi:10.17226/13139

3 Hillis S, Mercy J, Amobi A, et al. Global prevalence of past-year violence against children: a systematic review and minimum estimates. Pediatrics 2016;137:e20154079.

4 World Health Organization. Global and regional estimates of violence against women: prevalence and health effects of intimate partner violence and non-partner sexual violence, 2013.

5 Bott S, Guedes A, Ruiz-Celis AP, et al. Intimate partner violence in the Americas: a systematic review and reanalysis of national prevalence estimates. Rev Panam Salud Publica 2019;43:e26.

6 Coll CVN, Ewerling F, García-Moreno C, et al. Intimate partner violence in 46 low-income and middle-income countries: an appraisal of the most vulnerable groups of women using National health surveys. BMJ Glob Health 2020;5:e002208.

7 Fry DA, Elliott SP. Understanding the linkages between violence against women and violence against children. Lancet Glob Health 2017;5:e472-3.

8 Kyegombe N, Abramsky T, Devries KM, et al. What is the potentia for interventions designed to prevent violence against women to reduce children's exposure to violence? Findings from the SASA! study, Kampala, Uganda. Child Abuse Negl 2015;50:128-40.

9 Guedes A, Mikton C. Examining the intersections between child maltreatment and intimate partner violence. West $J$ Emerg Med 2013;14:377-9.
10 Bacchus LJ, Colombini M, Contreras Urbina M, et al. Exploring opportunities for coordinated responses to intimate partner violence and child maltreatment in low and middle income countries: a scoping review. Psychol Health Med 2017;22:135-65.

11 Hamby S, Finkelhor D, Turner $\mathrm{H}$. The overlap of witnessing partner violence with child maltreatment and other victimizations in a nationally representative survey of youth. Child Abus Neg/2010.

12 Gage AJ, Silvestre EA, violence M. Maternal violence, victimization, and child physical punishment in Peru. Child Abuse Negl 2010;34:523-33.

13 Chan KL. Children exposed to child maltreatment and intimate partner violence: a study of co-occurrence among Hong Kong Chinese families. Child Abuse Negl 2011;35:532-42.

14 Al-Eissa MA, Saleheen HN, Almuneef M. Examining the relationship between witnessing intimate partner violence and victimization among children in Saudi Arabia. J Interpers Violence 2020;35:1334-50.

15 Chan KL. Association between intimate partner violence and child maltreatment in a representative student sample in Hong Kong. Violence Against Women 2015;21:500-15.

16 Dalal K, Lawoko S, Jansson B. The relationship between intimate partner violence and maternal practices to correct child behavior: a study on women in Egypt. J Inj Violence Res 2010;2:25-33.

17 Shen AC-T. Long-Term effects of interparental violence and child physical maltreatment experiences on PTSD and behavior problems: a national survey of Taiwanese college students. Child Abuse Negl 2009;33:148-60.

18 Zolotor AJ, Theodore AD, Coyne-Beasley T, et al. Intimate partner violence and child maltreatment: overlapping risk. Brief Treat Crisis Interv 2007;7:305-21.

19 Guedes A, Bott S, Garcia-Moreno C. Violence Against Women and Violence Against Children - The Points of Intersection : Causes, Consequences and Solutions, 2016: 1-4.

20 Devries KM, Knight L, Child JC, et al. Witnessing intimate partner violence and child maltreatment in Ugandan children: a crosssectional survey. BMJ Open 2017;7:1-9.

21 Tajima EA. Risk factors for violence against children. $J$ Interpers Violence 2002;17:122-49.

22 Carlson C, Namy S, Norcini Pala A, et al. Violence against children and intimate partner violence against women: overlap and common contributing factors among caregiver-adolescent dyads. BMC Public Health 2020;20:124.

23 Moffitt TE, Arseneault L, Danese A, Klaus-Grawe 2012 Think Tank. Childhood exposure to violence and lifelong health: clinical intervention science and stress-biology research join forces. Dev Psychopathol 2013;25:1619-34.

24 Nunes AJ, Sales MCV. Violence against children in Brazilian scenery. Cien Saude Colet 2016;21:871-80.

25 Cardia N, Claudinei PL, Affonso C. Assessment of child maltreatment prevention readiness country report Brazil 2012. 70, 2012.

26 Schraiber LB, D'Oliveira AFPL, França-Junior I, et al. [Prevalence of intimate partner violence against women in regions of Brazil]. Rev Saude Publica 2007;41:797-807.

27 Viola TW, Salum GA, Kluwe-Schiavon B, et al. The influence of geographical and economic factors in estimates of childhood abuse and neglect using the childhood trauma questionnaire: a worldwide meta-regression analysis. Child Abuse Negl 2016;51:1-11.

28 Reichenheim ME, Dias AS, Moraes CL. Co-ocorrência de violência física conjugal E Contra filhos em serviços de saúde. Rev Saude Publica 2006;40:595-603.

29 Hallal PC, Bertoldi AD, Domingues MR, et al. Cohort profile: the 2015 Pelotas (Brazil) birth cohort study. Int J Epidemiol 2018;47:1048-1048h.

30 Garcia-Moreno C, Jansen H, Ellsberg M. WHO Multi-Country Study on Women's Health and Domestic Violence Against Women: Report on the First Results. World Heal Organ 2005:55-89.

31 Finkelhor D, Hamby S, Turner $\mathrm{H}$. The juvenile victimization questionnaire: 2nd revision (JVQ-R2. Durham, NH: Crimes Against Children Research Center, 2011. http://www.unh.edu/ccrc/jvq/ available_versions.html

32 Finkelhor D, Hamby SL, Ormrod R, et al. The juvenile victimization questionnaire: reliability, validity, and national norms. Child Abuse Negl 2005;29:383-412.

33 Universidade Federal do Rio Grande do Sul Furlan R dos S. Validação de conteúdo para a língua portuguesa do Juvenille victimization questionnaire. Porto Alegre, 2013. https://lume.ufrgs.br/ handle/10183/66654

34 da SRWS. Adaptação transcultural do juvenile victimization questionnaire R2 em amostra brasileira E mensuração de cortisol em crianças vítimas de Maus tratos. Pontifícia Universidade Católica do 
Rio Grande do Sul: Porto Alegre, 2017. http://tede2.pucrs.br/tede2/ handle/tede/7648

35 Yakubovich AR, Stöckl H, Murray J, et al. Risk and protective factors for intimate partner violence against women: systematic review and meta-analyses of Prospective-Longitudinal studies. Am J Public Health 2018;108:e1-11.

36 Milner J, Chilamkurti C. Physical child abuse perpetrator characteristics: a review of the literature. J Interpers Violence1991.

37 Schumacher JA, Slep AMS, Heyman RE. Risk factors for child neglect. Aggress Violent Behav 2001;6:231-54.

38 Mulder TM, Kuiper KC, van der Put CE, et al. Risk factors for child neglect: a meta-analytic review. Child Abuse Negl 2018;77:198-210.

39 Bidarra ZS, Lessard G, Dumont A. Co-Occurrence of intimate partner violence and child sexual abuse: prevalence, risk factors and related issues. Child Abuse Negl 2016;55:10-21.

40 Mujahid MS, Diez Roux AV, Morenoff JD, et al. Assessing the measurement properties of neighborhood scales: from psychometrics to ecometrics. Am J Epidemiol 2007;165:858-67.

41 Amorim P. Mini international neuropsychiatric interview (mini): validação de entrevista breve para diagnóstico de transtornos mentais. Rev Bras Psiquiatr 2000;22:106-15.

42 Sheehan DV, Lecrubier Y, Sheehan KH, et al. The Mini-International neuropsychiatric interview (M.I.N.I.): the development and validation of a structured diagnostic psychiatric interview for DSM-IV and ICD10. J Clin Psychiatry 1998;59 Suppl 20:22-33.

43 Amorim P, Lecrubier Y, Weiller E. DSM-III-R Psychotic Disorders : procedural validity of the Mini International Neuropsychiatric Interview (MINI). Eur Psychiatry 1998;13:26-34.

44 Caspi A, Taylor A, Smart M, et al. Can women provide reliable information about their children's fathers? cross-informant agreement about men's lifetime antisocial behaviour. J Child Psychol Psychiatry 2001;42:915-20

45 Hooley JM, Orley J, Teasdale JD. Levels of expressed emotion and relapse in depressed patients. Br J Psychiatry 1986;148:642-7.

46 Santos IS, Matijasevich A, Tavares BF, et al. Validation of the Edinburgh postnatal depression scale (EPDS) in a sample of mothers from the 2004 Pelotas birth cohort study. Cad Saude Publica 2007;23:2577-88.

47 WHO ASSIST Working Group. The alcohol, smoking and substance involvement screening test (assist): development, reliability and feasibility. Addiction 2002;97:1183-94.
48 Henrique IFS, De Micheli D, Lacerda RBde, et al. [Validation of the Brazilian version of Alcohol, Smoking and Substance Involvement Screening Test (ASSIST)]. Rev Assoc Med Bras 2004;50:199-206.

49 Barros AJD, Hirakata VN. Alternatives for logistic regression in crosssectional studies: an empirical comparison of models that directly estimate the prevalence ratio. BMC Med Res Methodol 2003;3:21.

50 Victora CG, Huttly SR, Fuchs SC, et al. The role of conceptual frameworks in epidemiological analysis: a hierarchical approach. Int J Epidemiol 1997;26:224-7.

51 Devries K, Merrill KG, Knight L, et al. Violence against children in Latin America and the Caribbean: what do available data reveal about prevalence and perpetrators? Rev Panam Salud Pública 2019;43:1.

52 Appel AE, Holden GW. The co-occurrence of spouse and physical child abuse: a review and appraisal. J Fam Psychol 1998;12:578-99.

53 Daly M, Wilson M. Child abuse and other risks of not living with both parents. Ethol Sociobiol 1985.

54 Berger LM, Carlson MJ, Bzostek SH, et al. Parenting practices of resident fathers: the role of marital and biological ties. J Marriage Fam 2008;70:625-39.

55 Petren RE, Garneau-Rosner CL, Yildirim ED. Union stability among mothers and stepfathers: contributions of stepfathers and biological fathers. J Fam Psychol 2018;32:1142-51.

56 Alexandre GC, Nadanovsky P, Moraes CL, et al. The presence of a stepfather and child physical abuse, as reported by a sample of Brazilian mothers in Rio de Janeiro. Child Abuse Negl 2010;34:959-66

57 Jaffee SR, Moffitt TE, Caspi A, et al. Life with (or without) father: the benefits of living with two biological parents depend on the father's antisocial behavior. Child Dev 2003;74:109-26.

58 Choi KW, Houts R, Arseneault L. Maternal depression in the intergenerational transmission of childhood maltreatment and its sequelae: testing postpartum effects in a longitudinal birth cohort. Dev Psychopathol 2018;31:1-14.

59 Madigan S, Cyr C, Eirich R, et al. Testing the cycle of maltreatment hypothesis: meta-analytic evidence of the intergenerational transmission of child maltreatment. Dev Psychopathol 2019;31:23-51.

60 Olds DL, Sadler L, Kitzman H. Programs for parents of infants and toddlers: recent evidence from randomized trials. $J$ Child Psychol Psychiatry 2007;48:355-91. 